\title{
Simple mathematical approach to solar cell/panel behavior based on datasheet information
}

\author{
Santiago Pindado*, Javier Cubas \\ Instituto Universitario de Microgravedad "Ignacio Da Riva"(IDR/UPM), Universidad Politécnica de Madrid, ETSI Aeronáutica y del Espacio, Pza. del Cardenal \\ Cisneros 3, Madrid, 28040, Spain
}

\section{A R T I C L E I N F O}

\section{Article history:}

Received 16 March 2016

Received in revised form

12 October 2016

Accepted 2 November 2016

Available online $\mathrm{xxx}$

\section{Keywords:}

Solar cell

Photovoltaic array modeling

Circuit model

Parameter extraction

\begin{abstract}
A B S T R A C T
A new explicit mathematical expression is used to describe the behavior of a photovoltaic device (solar cell/panel), that is, its $I-V$ curve, based on the characteristic points normally included in the manufacturers' datasheets. This expression consists of two simple equations, one for voltage levels lower than the voltage at the maximum power point, $V<V_{m p}$, and the other one for voltage levels above this point, $V>V_{m p}$. The first equation is defined with two of the three characteristic points (short circuit and maximum power points), whereas the second one is defined with the current and voltage levels at maximum power point, the open circuit voltage, and a constant that can be adjusted based on: 1) the best fitting to the data within the bracket $\left[V_{m p}, V_{o c}\right]$, or 2) one point within this bracket, or 3 ) the slope of the $I-V$ curve at the open circuit point, or 4 ) an estimation of that slope. Results of the solar cell/panel behavior analysis obtained with the proposed methodology, are similar to the ones obtained with the well-known 1-diode/2-resistor equivalent circuit model, in terms of accuracy.
\end{abstract}

\section{Introduction}

Within the last decades, the use of photovoltaic devices (solar cells/panels) has been greatly increased as a clean way to obtain energy, or due to its obvious advantages in autonomous systems such as the ones designed for space operations (i.e., satellites, spacecraft).

Today, the most common way to simulate the behavior of such photovoltaic devices is through equivalent circuits. See in Fig. 1 the 1-diode/2-resistor equivalent circuit model of a solar cell/panel whose behavior (that is, the relationship between the output current, $I$, and the output voltage, $V$ ), is defined by the following implicit equation:

$I=I_{p v}-I_{D 1}-\frac{V+I R_{S}}{R_{s h}}=I_{p v}-I_{0}\left[\exp \left(\frac{V+I R_{S}}{n a V_{T}}\right)-1\right]-\frac{V+I R_{S}}{R_{s h}}$

where $R_{S}$ and $R_{S h}$ are the series and shunt resistors, $I_{D 1}$ is the current through the diode, $I_{p v}$ is the photocurrent delivered by the current source, $I_{0}$ is the saturation current of that diode, $V_{T}$ is the thermal voltage (defined as a function of the temperature, the charge of the electron, and the Boltzmann constant), $a$ is ideality factor of the diode, and $n$ is the number of series-connected cells in the device. More information on this model can be found at $[1,2]$. The main problem when using the equivalent circuit consists in the parameter extraction, that is, all of the above parameters need to be correctly estimated in order to obtain a good fitting to the currentvoltage $I-V$ curve of the solar cell/panel (an example of this curve is shown in Fig. 1).

There are multiple procedures (analytical, computational) to extract the equivalent circuit parameters depending on the information available in relation to the photovoltaic device. This information can be the $I-V$ curve measured experimentally or, in most cases, the current and voltage levels at the characteristic points which can be found in the manufacturer's datasheets (short circuit current, $I_{s c}$, maximum power current and voltage, $I_{m p}$ and $V_{m p}$, and open circuit voltage, $V_{o c}$ ). In Refs. [3-8] a thorough review of the different methods for fitting the equivalent circuit to the solar cell/ panel behavior can be found. The equivalent circuit parameter extraction process is not an immediate task, and even doing it by analytical methods it requires a quite large number of calculations $[1,2]$. Some efforts have been made in order to ease the parameter extraction procedure, the use of a reduced number of points of the 

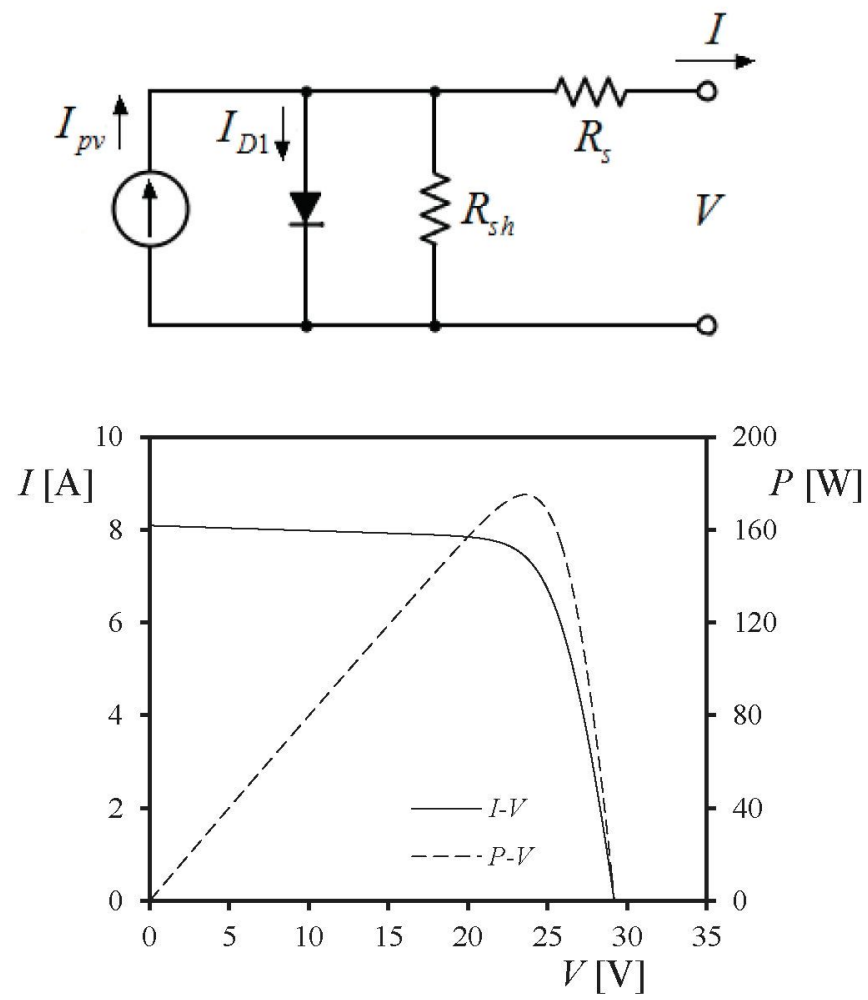

Fig. 1. Top: 1-diode/2-resistor equivalent circuit model of a solar cell/panel. Bottom: $I_{-}$ $V$ and $P$ - $V$ curves of a Kyocera KC175GHT-2 solar panel at constant solar irradiance $\left(1000 \mathrm{~W} / \mathrm{m}^{2}\right)$ and $T=25^{\circ} \mathrm{C}$. This results were calculated using Equation (1) with $n a=48.548, I_{p v}=8.11 \mathrm{~A}, I_{0}=4.86 \cdot 10^{-10} \mathrm{~A}, R_{s}=0.262 \Omega$, and $R_{s h}=90.85 \Omega$.

$I-V$ curve being an interesting methodology [9-11], together with the use of expressions based on the fill factor [12-14].

In the present paper, a new simple mathematical method to approach the behavior of a photovoltaic device is proposed as an alternative to the equivalent circuit models. In some applications a very quick and easy approach to a solar cell/panel behavior is required. We have detected this need when programming the power subsystem module of the Concurrent Design Facility (CDF) for space missions at the IDR/UPM Institute. Within this module, the solar panels behavior has to be calculated in a quite large number of different situations, including sometimes real-time programing on spreadsheets. In this specific case, once a proper level of accuracy is guaranteed, the simpler methodology is preferred. This work is part of a larger research framework carried out at the IDR/UPM Institute to analyze photovoltaic systems behavior, in order to produce models to be involved in more complex simulations developed to study space systems $[1,2,15-18]$.

Furthermore, some professionals from the photovoltaic sector may not have the resources (human or technical), to program equivalent circuit models in order to optimize their energy systems. Based in our experience, this could happen at the first steps of a project (that is, the pre-design for engineering tenders/proposals), which are normally characterized by a strict limitation on time and expenses.

Some examples of $I-V$ curve explicit expressions can be found in the literature. For instance, Akbaba and Alattawi suggested a model for the solar cell/panel behavior based on the following equation [19]:

$I=\frac{V_{o c}-V}{A+B V^{2}-C V}$

where $A, B$ and $C$, are constants that depend on the short circuit current, the open circuit voltage, and two additional points of the $I$ $V$ curve. A model based on a similar equation has been recently proposed by Miceli et al. [20]. El-Tayyan proposed a simpler equation, only dependent on two coefficients, $C_{1}$ and $C_{2}$ [21]:

$I=I_{s c}-C_{1} \exp \left(-\frac{V_{o c}}{C_{2}}\right)\left[\exp \left(\frac{V}{C_{2}}\right)-1\right]$

that can be either estimated or calculated based on the characteristic points of the $I-V$ curve [22]. Besides, polynomials fitted to the $I-$ $V$ curve have been also proposed to describe the solar cell/panel performances [23,24]. An even simpler model is the power law model suggested by Karmalkar and Haneefa $[25,26]$ :

$\frac{I}{I_{S C}}=1-(1-\gamma) \frac{V}{V_{o c}}-\gamma\left(\frac{V}{V_{o c}}\right)^{m}$,

where constants $\gamma$ and $m$ are calculated with the current and voltage levels at points $V / V_{o c}=0.6$ and $I / I_{s c}=0.6$, respectively. Another power law relation between solar cell/panel current and voltage output levels were independently proposed by Das [27] and Saetre et al. [28]:

$\frac{I}{I_{s c}}=\left[1-\left(\frac{V}{V_{o c}}\right)^{f}\right]^{\frac{1}{g}}$,

where coefficients $f$ and $g$ are estimated with output current measurements at $V / V_{o c}=0.8$ and $V / V_{o c}=0.9$. Finally, Das also proposed the equation:

$\frac{I}{I_{S C}}=\frac{1-\left(\frac{V}{V_{o c}}\right)^{k}}{1+h\left(\frac{V}{V_{o c}}\right)}$,

as a suitable model to describe solar cell/panel performance, $h$ and $k$ being calculated with the slope of the $I-V$ curve at short circuit and open circuit points [29].

The model proposed in this paper is based on the shape of the power curve (i.e., output power in relation to the output voltage) of the solar/cell panel (see in the graph of Fig. 1 an example of this power curve). This $P-V$ curve has been modelled sometimes through a third-degree polynomial, in order to obtain the voltage level of maximum power point in photovoltaic systems [30]. In the present work, the $P-V$ curve is modelled with two different simple mathematical equations, one for voltage levels lower than the maximum power voltage, $V_{m p}$ (that is, within the bracket $\left[0, V_{m p}\right]$ ), and another one for voltage levels above that maximum power voltage (within the bracket $\left[V_{m p}, V_{o c}\right]$ ). Once the equations for the output power are defined, the output current can be easily derived by dividing by the output voltage. As a result, an easy and explicit expression of the output current as a function of the output voltage is derived.

The present work is organized as follows. In Section 2 the proposed model is described, whereas in Section 3 the model validation is carried out by its application to different solar cells/panels and comparison to the 1-diode/2-resistor equivalent circuit model. Finally, the conclusions are summarized in Section 4.

\section{Equations proposed}

As said in the first section, the approach to the experimental data related to a photovoltaic device is done with different equations depending on the considered bracket. If the first bracket, [0, $\left.V_{m p}\right]$, is considered, the proposed equation for the power is: 
$P=a V\left(V^{\xi}-b\right)$

where $P$ is the power supplied by the solar cell/panel, $V$ is the output voltage, and $a, b$ and $\xi$ are constants to be adjusted. Three conditions are needed in order to extract the proper values of these constants. In the first place, the value of the power divided by the voltage at the short circuit point has to be equal to the short circuit current, $I_{s c}$, and therefore:

$\lim _{V \rightarrow 0} \frac{P}{V}=-a b=I_{S C}$

then, from the condition at the maximum power point, $\partial P / \partial V=0$, it is possible to derive the following equation:

$b=(1+\xi) V_{m p}^{\xi}$

where $V_{m p}$ is the voltage at the maximum power point. Besides, from Equations (7) and (9) and evaluating the maximum power at this point:

$\left.P\right|_{V=V_{m p}}=V_{m p} I_{m p}=-a \xi V_{m p}^{\xi+1}$

therefore:

$a=-\frac{I_{m p}}{\xi V_{m p}^{\xi}}$

In addition, an expression for constant $\xi$ can be derived from Equations (8), (9) and (11):

$\xi=\frac{I_{m p}}{I_{S C}-I_{m p}}$.

Finally, as both constants $a$ and $b$ can be exclusively expressed in relation to the third one, $\xi$, Equation (7) can be expressed in terms of the characteristic points of the $I-V$ curve:

$P=I_{S C} V\left[1-\left(1-\frac{I_{m p}}{I_{S c}}\right)\left(\frac{V}{V_{m p}}\right)^{\frac{I_{m p}}{I_{S c}-I_{m p}}}\right]$

Consequently, an expression for the solar cell/panel behavior in the first bracket, which is only dependent on $I-V$ curve characteristic points is obtained.

On the other hand, the proposed equation for the second bracket, $\left[V_{m p}, V_{o c}\right]$, is:

$P=V_{m p} I_{m p}\left[1-\left(\frac{V-V_{m p}}{V_{o c}-V_{m p}}\right)^{\eta}\right] ; \eta>1$,

where $V_{o c}$ is the open circuit voltage and $\eta$ is a constant to be adjusted. The value of constant $\eta$ can be derived from the slope of the power at the open circuit point, taking into account that:

$\left.\frac{\partial P}{\partial V}\right|_{V=V_{o c}}=\left.\frac{\partial I}{\partial V}\right|_{V=V_{o c}} V_{o c}=-\eta \frac{V_{m p} I_{m p}}{V_{o c}-V_{m p}}$,

which leads to:

$\eta_{s l}=-\left.\frac{\partial I}{\partial V}\right|_{V=V_{o c}} \frac{V_{o c}}{I_{m p}}\left(\frac{V_{o c}}{V_{m p}}-1\right)$.

In the above expression, the slope of the $I-V$ curve can be extracted from the experimental data, or estimated:

1) using the empirical expression defined by Orioli and Gangi [31]:

$R_{S 0}=-\left.\frac{\partial V}{\partial I}\right|_{V=V_{o c}}=C_{S} \frac{V_{o c}}{I_{S C}}$

with $C_{S}=0.11175$ (based on the photovoltaic panels' behavior studied by the aforementioned authors), that leads to:

$\eta_{e s i 1}=\frac{1}{C_{S}} \frac{I_{S C}}{I_{m p}}\left(\frac{V_{o c}}{V_{m p}}-1\right)$

2) or using another interesting estimation of the slope of the $I-V$ curve at the open circuit point derived from the work of Deihimi et al. [32]. Based on this work, the following expression can be assumed:

$\left.\frac{\partial I}{\partial V}\right|_{V=V_{o c}} \cong\left(\frac{I_{S C}}{V_{o c}}\right)^{2} \frac{1}{\left.\frac{\partial I}{\partial V}\right|_{V=0}}$,

which, if the following approach is accepted as reasonable:

$\left.\frac{\partial I}{\partial V}\right|_{V=0} \cong \frac{I_{m p}-I_{S C}}{V_{m p}}$

leads to the following estimation of $\eta$ :

$\eta_{e s i 2}=\frac{I_{S C}}{I_{m p}}\left(\frac{I_{S c}}{I_{s c}-I_{m p}}\right)\left(\frac{V_{o c}-V_{m p}}{V_{o c}}\right)$.

Besides, the value of constant $\eta$ can also be derived from the best fitting to the experimental data from the second bracket $\left[V_{m p}, V_{o c}\right]$, in terms of Normalized Root Mean Squared Error (NRMSE), hereinafter referred as $\varepsilon$ :

$\varepsilon=\sqrt{\left.\frac{1}{N} \sum_{j=1}^{N} \frac{I_{c a l c, j}-I_{j}}{I_{j}}\right)^{2}}$,

where $I$ stands for the current at the reference $I-V$ curve, and $I_{\text {calc }}$ stands for the calculated points at the same voltage levels. $N$ is the number of points from the reference $I-V$ curve within the bracket. Constant $\eta$ was estimated in the present work with this procedure (i.e., minimizing error $\varepsilon$ ), in order to compare with the results obtained by the other methodologies explained above. The figures obtained by this optimization are denoted as $\eta_{b f}$ hereinafter. Finally, another estimation of $\eta$ can be obtained with the voltage and current values $V^{*}$ and $I^{*}$, at one point within that second bracket:

$\eta_{p}=\frac{\ln \left(V_{m p} I_{m p}-V^{*} I^{*}\right)-\ln \left(V_{m p} I_{m p}\right)}{\ln \left(V^{*}-V_{m p}\right)-\ln \left(V_{o c}-V_{m p}\right)}$.

From the above equations it is possible to derive a general expression, consisting in two explicit equations, for the output current of a solar cell/panel in relation to the output voltage: 


$$
I= \begin{cases}I_{s C}\left[1-\left(1-\frac{I_{m p}}{I_{s c}}\right)\left(\frac{V}{V_{m p}}\right)^{\left.\frac{I_{m p}}{I_{s c}-I_{m p}}\right]}\right. & ; V \leq V_{m p} \\ I_{m p} \frac{V_{m p}}{V}\left[1-\left(\frac{V-V_{m p}}{V_{o c}-V_{m p}}\right)^{\eta}\right] & ; V \geq V_{m p}\end{cases}
$$

with constant $\eta$ estimated (Equations (18), (21)), calculated if additional information is available (Equation (16) or (23)), or extracted from the best fitting to the $I-V$ curve within the bracket $\left[V_{m p}, V_{o c}\right]$.

It should also be mentioned that the above equations have the same slope at the maximum power point, that is:

$$
\begin{aligned}
\left.\frac{\partial}{\partial V}\left[a\left(V^{\xi}-b\right)\right]\right|_{V=V_{m p}^{-}} & =\left.\frac{\partial}{\partial V}\left\{\frac{V_{m p} I_{m p}}{V}\left[1-\left(\frac{V-V_{m p}}{V_{o c}-V_{m p}}\right)^{\eta}\right]\right\}\right|_{V=V_{m p}^{+}} \\
& =-\frac{I_{m p}}{V_{m p}}
\end{aligned}
$$

which matches the mathematical equation of the slope of the $I-V$ curve at the maximum power point:

$$
\begin{aligned}
\left.\frac{\partial P}{\partial V}\right|_{V=V_{m p}} & =0=\left.\frac{\partial V I}{\partial V}\right|_{V=V_{m p}}=I_{m p}+\left.\left.\frac{\partial I}{\partial V}\right|_{V=V_{m p}} V_{m p} \Rightarrow \frac{\partial I}{\partial V}\right|_{V=V_{m p}} \\
& =-\frac{I_{m p}}{V_{m p}} .
\end{aligned}
$$

\section{Model validation}

\subsection{Comparison to the 1-diode/2-resistor model}

The model validation has been firstly carried out by comparison to the well-known 1-diode/2-resistor equivalent circuit model (i.e., Equation (1)). In order to compare both mathematical expressions, dimensionless variables are used: output current, $i=I / I_{s c}$, and output voltage, $v=V / V_{o c}$. The proposed model is then rewritten as:

$$
i=\left\{\begin{array}{ll}
1-(1-\beta)\left(\frac{v}{\alpha}\right)^{\frac{\beta}{1-\beta}} & ; v \leq \alpha \\
\frac{\alpha}{v}\left[1-\left(\frac{v-\alpha}{1-\alpha}\right)^{\left.\frac{1-\alpha}{\beta(1-\beta)}\right]}\right. & ; v \geq \alpha
\end{array},\right.
$$

where $\alpha=V_{m p} / V_{o c}$ and $\beta=I_{m p} / I_{s c}$, are the coordinates of the maximum power point, and coefficient $\eta$ in Equation (24) is calculated with Equation (21). On the other hand, Equation (1), corresponding to the 1 -diode/2-resistor model, can be rewritten as:

$i=i_{p v}-i_{0}\left[\exp \left(\frac{v+i r_{s}}{\lambda}\right)-1\right]-\frac{v+i r_{s}}{r_{s h}}$,

where now the five non-dimensional parameters to be extracted are $i_{p v}=I_{p v} / I_{s c}, i_{0}=I_{0} / I_{s c}, \lambda=n a V_{T} / V_{o c}, r_{s}=R_{s} I_{s c} / V_{o c}$, and $r_{s h}=R_{s h} I_{s c}$ $V_{o c}$.

In order to extract the five parameters of Equation (28) from the four conditions related to the solar cell/panel characteristic points
Table 1

Characteristic points $\left(I_{s c}, I_{m p}, V_{m p}\right.$, and $\left.V_{o c}\right)$ of the studied solar cells/panels.

\begin{tabular}{llllll}
\hline Solar cell/panel & Technology & $I_{s c}[\mathrm{~A}]$ & $I_{m p}[\mathrm{~A}]$ & $V_{m p}[\mathrm{~V}]$ & $V_{o c}[\mathrm{~V}]$ \\
\hline RTC France & $\mathrm{Si}$ & 0.7605 & 0.6894 & 0.4507 & 0.5727 \\
TNJ Spectrolab & GalnP2/GaAs/Ge & 0.5239 & 0.4960 & 2.270 & 2.565 \\
ZTJ Emcore & InGaP/InGaAs/Ge & 0.4628 & 0.4389 & 2.410 & 2.726 \\
Azur Space 3G30C & GalnP/GaAs/Ge & 0.5202 & 0.5044 & 2.411 & 2.70 \\
Photowatt PWP 201 & $\mathrm{Si}$ & 1.032 & 0.9255 & 12.493 & $\mathbf{1 6 . 7 7 8}$ \\
Kyocera KC200GT-2 & $\mathrm{Si}$ polycrystalline & 8.210 & 7.610 & 26.30 & 32.90 \\
Selex Galileo SPVS X5 & GalnP/GaAs/Ge & 0.50344 & 0.48476 & 12.099 & $\mathbf{1 3 . 5 7 5}$ \\
\hline
\end{tabular}

(three points and the slope of the $I-V$ curve at one of them), one more condition is required. The slope at open circuit point:

$r_{s 0}=-\left.\frac{\partial v}{\partial i}\right|_{v=1}=\frac{1}{-\left.\frac{\partial i}{\partial v}\right|_{\nu=1}}$,

can be used, as from the work by Phang et al. [33] it is possible to derive an equation that relates parameters $r_{s}$ and $\lambda$ with the slope of the non-dimensional $I-V$ curve (hereinafter, the $i$ - $v$ curve):

$r_{s} \approx r_{s 0}-\lambda \exp \left(-\frac{1}{\lambda}\right)$

The above expression can be combined with the following ones (from Ref. [2]):

$\frac{\lambda \alpha(2 \beta-1)}{(\alpha+\beta-1)\left(\alpha-\beta r_{s}\right)-\lambda(\alpha-\beta)}=\exp \left(\frac{\alpha+\beta r_{s}-1}{\lambda}\right)$

$r_{s h}=\frac{\left(\alpha-\beta r_{s}\right)\left(\alpha-(1-\beta) r_{s}-\lambda\right)}{\left(\alpha-\beta r_{s}\right)(1-\beta)-\lambda \beta}$

$i_{0}=\frac{r_{s h}+r_{s}-1}{r_{s h} \exp \left(\frac{1}{\lambda}\right)}$,

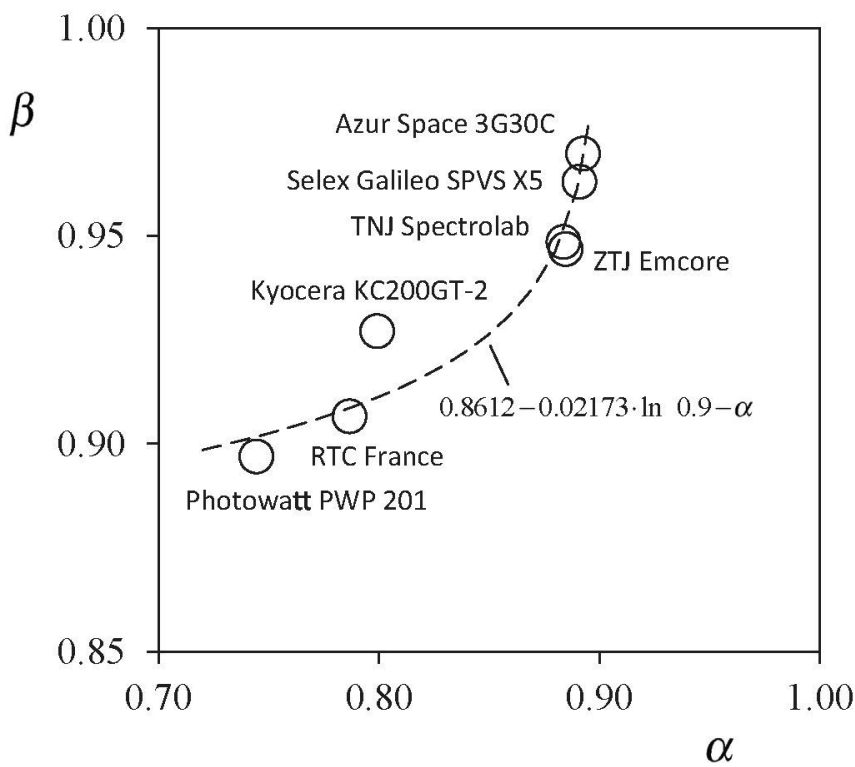

Fig. 2. Non-dimensional output voltage and current levels at maximum power, respectively $\alpha$ and $\beta$, for the different solar cell/panels studied in the present work (see also Table 1) 


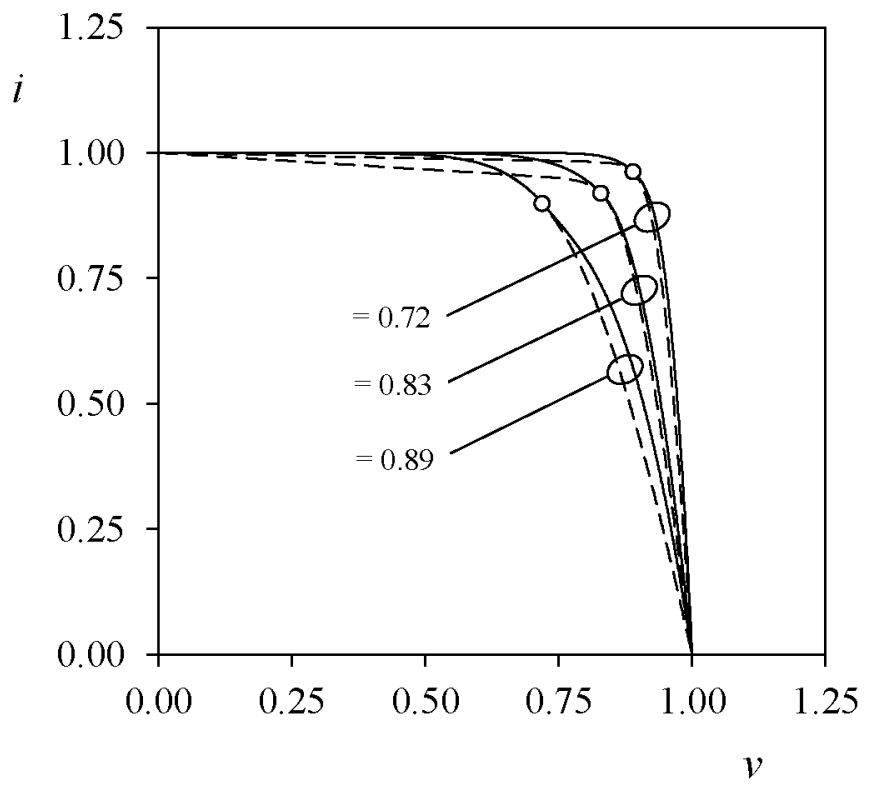

Fig. 3. Solid lines: $i-v$ curves calculated with the proposed method at $\alpha=0.72,0.83$ and 0.89 , and $\beta$ calculated with Equation (35). The maximum power points are indicated with open circles. Dashed lines: corresponding $i-v$ curves calculated with the 1diode/2-resistor equivalent circuit model (Equation (28)).

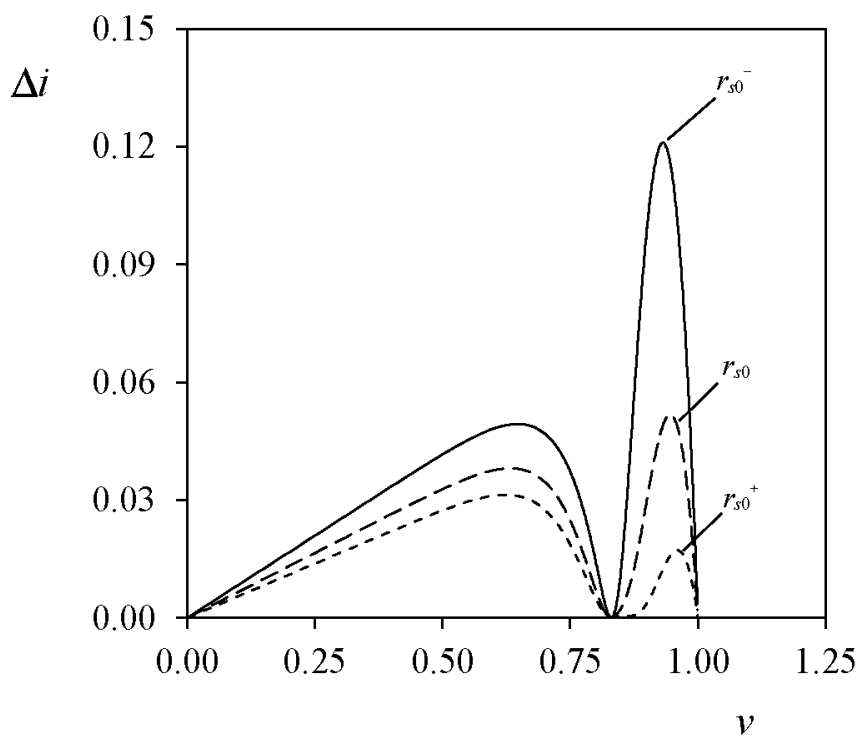

Fig. 4. Difference between the non-dimensional current, $\Delta i$, calculated with the present model and the 1-diode/2-resistor model for the case $\alpha=0.83 .1$-diode/2-resistor model calculated in three subcases: the initial slope at open circuit point equal to the one resulting by the present model (that is, $r_{s 0}=0.09787$ ) and this slope increased and decreased by $30 \%$ (subcases $r_{s 0}^{+}$and $r_{s 0}^{-}$).

$i_{p v}=1+\frac{r_{s}}{r_{s h}}$

to obtain the five parameters of expression (28) and, therefore, the $i-v$ curve, as a function of an initial estimation of the slope at open circuit point.

In Table 1, the characteristic points of seven photovoltaic solar cells/panels of different technologies (four solar cells, two solar panels, and one solar module for space applications) are included.
The coordinates $[\alpha, \beta]$ of the maximum power point related to each one of these photovoltaic devices are shown in Fig. 2. It can be observed in the figure that the following mathematical expression:

$\beta=0.8612-0.02173 \ln (0.9-\alpha)$

fits these points quite well. Bearing in mind this result, nineteen different $i-v$ curves were proposed to compare the present model to the 1-diode/2-resistor equivalent circuit model, its nondimensional output voltage level at maximum power, $\alpha$, ranging from $\alpha=0.72$ to $\alpha=0.895$, and the non-dimensional output current level at maximum power, $\beta$, defined with Equation (35).

In Fig. 3, three $i-v$ curves calculated with the proposed model (Equation (27)) with $\alpha=0.72,0.83$ and 0.89 , and $\beta$ based on Equation (35) are shown (solid lines). The corresponding curves based on the 1-diode/2-resistor equivalent circuit model are also shown (dashed lines), the value of $r_{50}$ in each of these three cases being based on the slope from the curves obtained with the proposed method. In the graph, the differences between both models can be appreciated. If the initial values given to $r_{s 0}\left(r_{s 0}=0.1414\right.$ $(\alpha=0.72), 0.09787(\alpha=0.83)$, and $0.04385(\alpha=0.89))$ are compared to the ones calculated with the slope from the resulting curves after the parameter extraction $\left(r_{s 0}=0.2084,0.1218\right.$, and 0.06162 , respectively), some limitations on the approximation proposed for the calculation of $r_{s}$ (Equation (30)) are revealed.

As a consequence, it seems that a better correlation between the proposed model and the 1-diode/2-resistor equivalent circuit model could be achieved if the initial values of $r_{s 0}$ are modified when calculating the parameters of Equation (28). In Fig. 4, the differences between the non-dimensional current, $\Delta i$, calculated with both models for the case $\alpha=0.83$, with the initial slope at open circuit point (that is, $r_{s 0}=0.09787$ ) and this slope increased and decreased by $30 \%$ (subcases $r_{s 0}^{+}$and $r_{s 0}^{-}$), are shown. A better correlation between models is clearly observed in the subcase $r_{s 0}^{+}$, the slope of the $i-v$ curve calculated with Equation (28), $d i$ / $\partial v=-9.2872$, more similar to the one from the proposed model, $\partial i$ / $\partial v=-10.2173$, than the resulting from the curves calculated in the other two subcases, $\partial i / \partial v=-6.6636\left(r_{s 0}^{-}\right)$and $\partial i / \partial v=-8.2131\left(r_{s 0}\right)$. In addition, the root mean squared difference between $i-v$ curves from the proposed method and the 1-diode/2-resistor equivalent circuit model:

$\sigma=\sqrt{\frac{1}{N} \sum_{j=1}^{N} \Delta i_{j}^{2}}$,

was calculated for the nineteen studied cases within the bracket $\alpha=0.72$ to $\alpha=0.895$ (in the above equation $N$ stands for the number of points that define the aforementioned $i-v$ curves). In Fig. 5 the results are shown, indicating that $i-v$ curves from the equivalent circuit model calculated with the increased slope $\left(r_{s 0}^{+}\right)$ have better correlation with the proposed method, accordingly to the result observed for $\alpha=0.83$. Furthermore, the correlation between both models is better from $\alpha=0.83$ to $\alpha=0.87$, it becoming worse for $\alpha \rightarrow 0.9$.

Some final comments can be added in relation to the series and shunt resistor values, resulting from the 1-diode/2-resistor equivalent circuit model fitted to the proposed model. First of all, the shunt resistor does not exist (i.e., $r_{s h} \rightarrow \infty$ ), as the slope of the $i-v$ curve at $i=0$ from the proposed model is zero. This fact makes the present method equivalent to the well-know 1-diode/1-resistor model [34]. Secondly, taking into account Equation (30) and the slope of the proposed method at open circuit point from Equation (16), it is possible to obtain a simple equation that relates the series resistor to the $\eta$ parameter: 


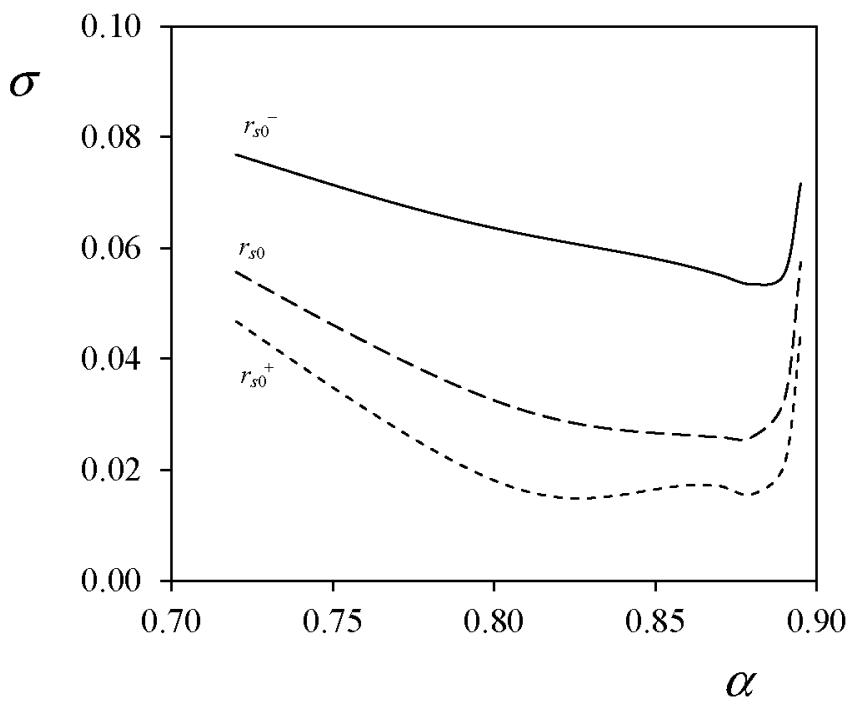

Fig. 5. Root mean squared difference between the non-dimensional current, $\Delta i$, calculated with the present model and the 1-diode/2-resistor model for $\alpha=0.72$ to $\alpha=0.895$. 1-diode/2-resistor model calculated in three subcases: the initial slope at open circuit point equal to the one resulting by the present model (that is, $r_{s 0}=0.09787$ ) and this slope increased and decreased by $30 \%$ (subcases $r_{50}^{+}$and $r_{50}^{-}$).
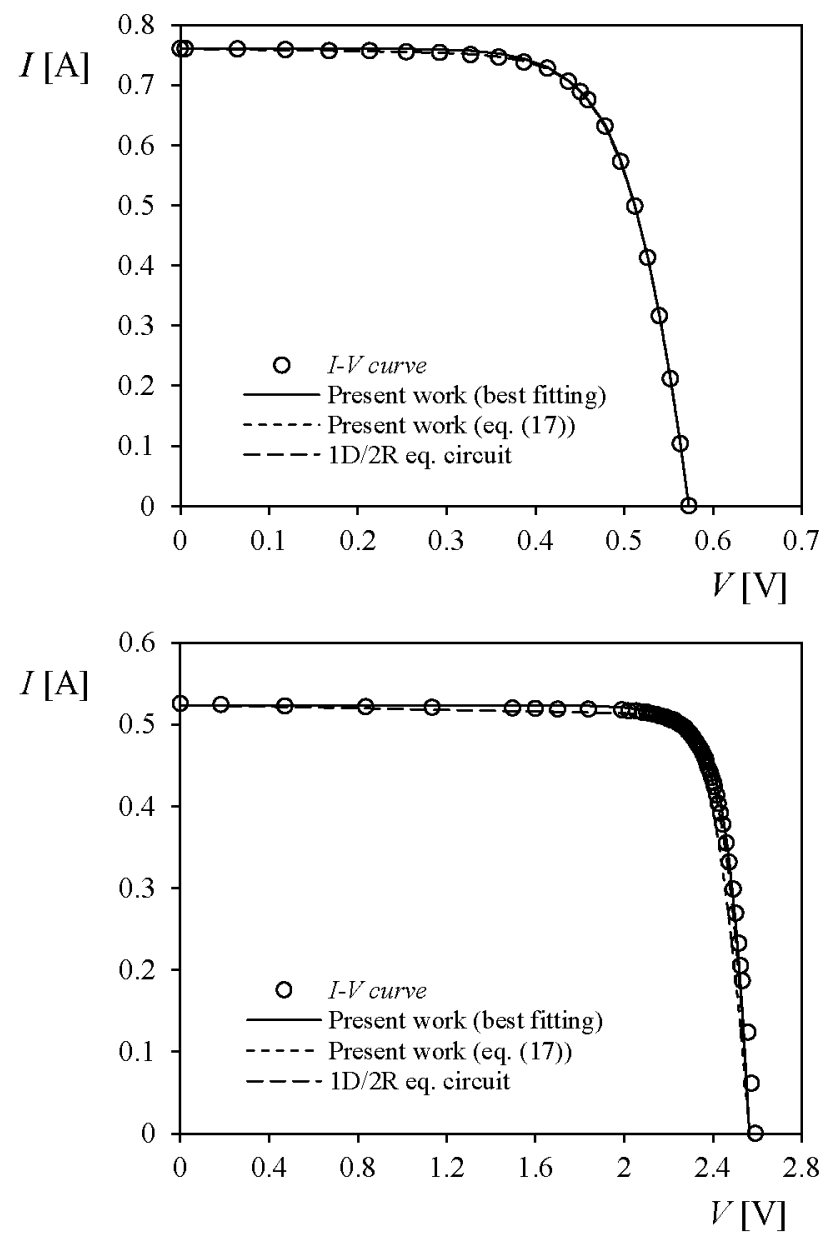

$r_{s} \approx \frac{1}{\eta}\left(\frac{1-v_{p m}}{v_{p m} i_{p m}}\right)-\lambda \exp \left(-\frac{1}{\lambda}\right)$

The above equation also relates the series resistor to the ideality factor, $a$, through the $\lambda$ parameter, this relationship being already suggested by Araujo and Sanchez for the 1-diode/1-resistor model [35]:

$r_{s}=2(1-\psi-\lambda)$,

where the new parameter $\psi$ is defined as:

$\psi=\int_{0}^{1} i \mathrm{~d} v$.

\subsection{Validation of the model in relation to experimental data}

Once the proposed model has been directly compared to the 1diode/2-resistor model, revealing in which cases (non-dimensional voltage at maximum power from $\alpha=0.83$ to $\alpha=0.87$ ) both models could have a better correlation, the model was used to modeling
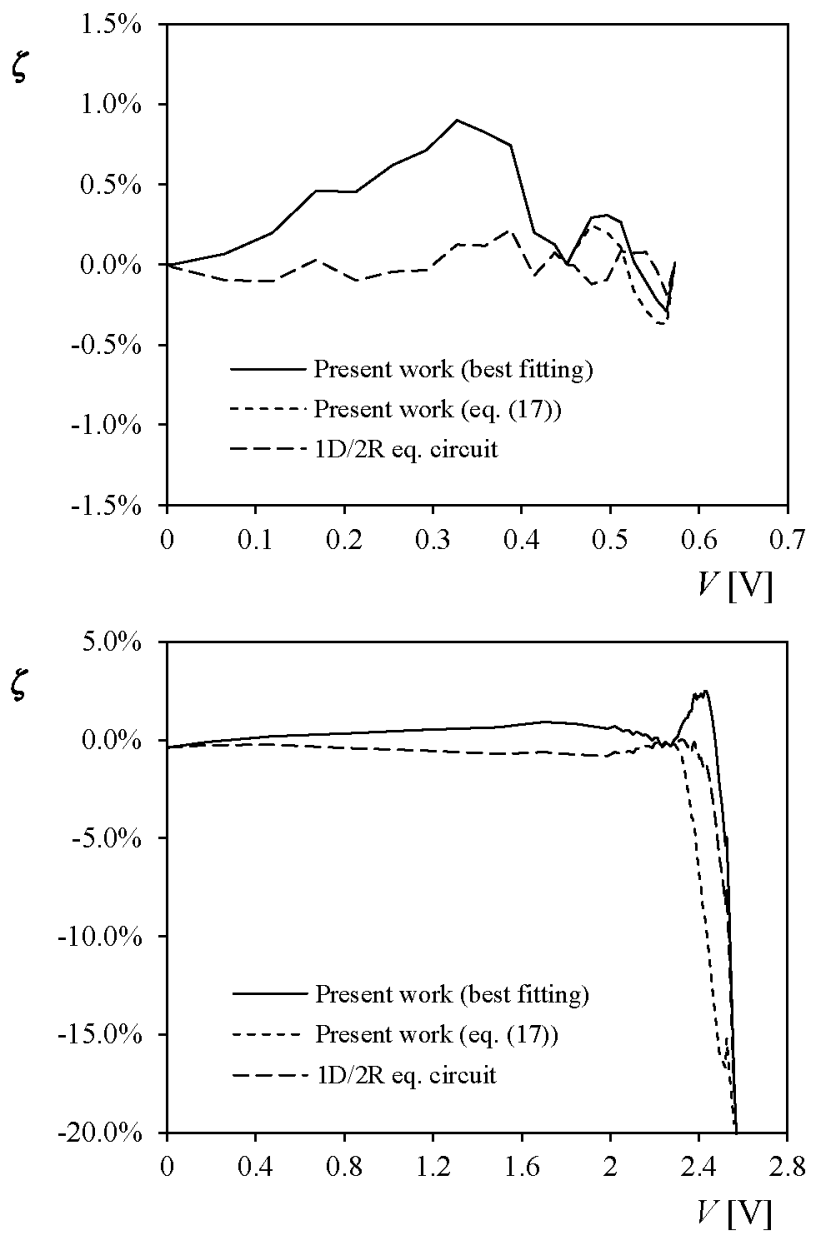

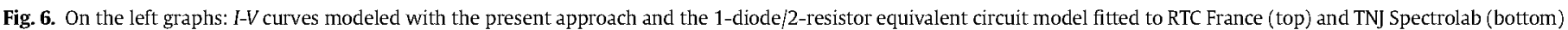

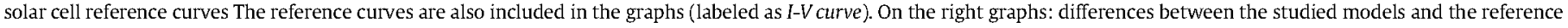
curves, made non dimensional with the short circuit current (Equation (40)). 

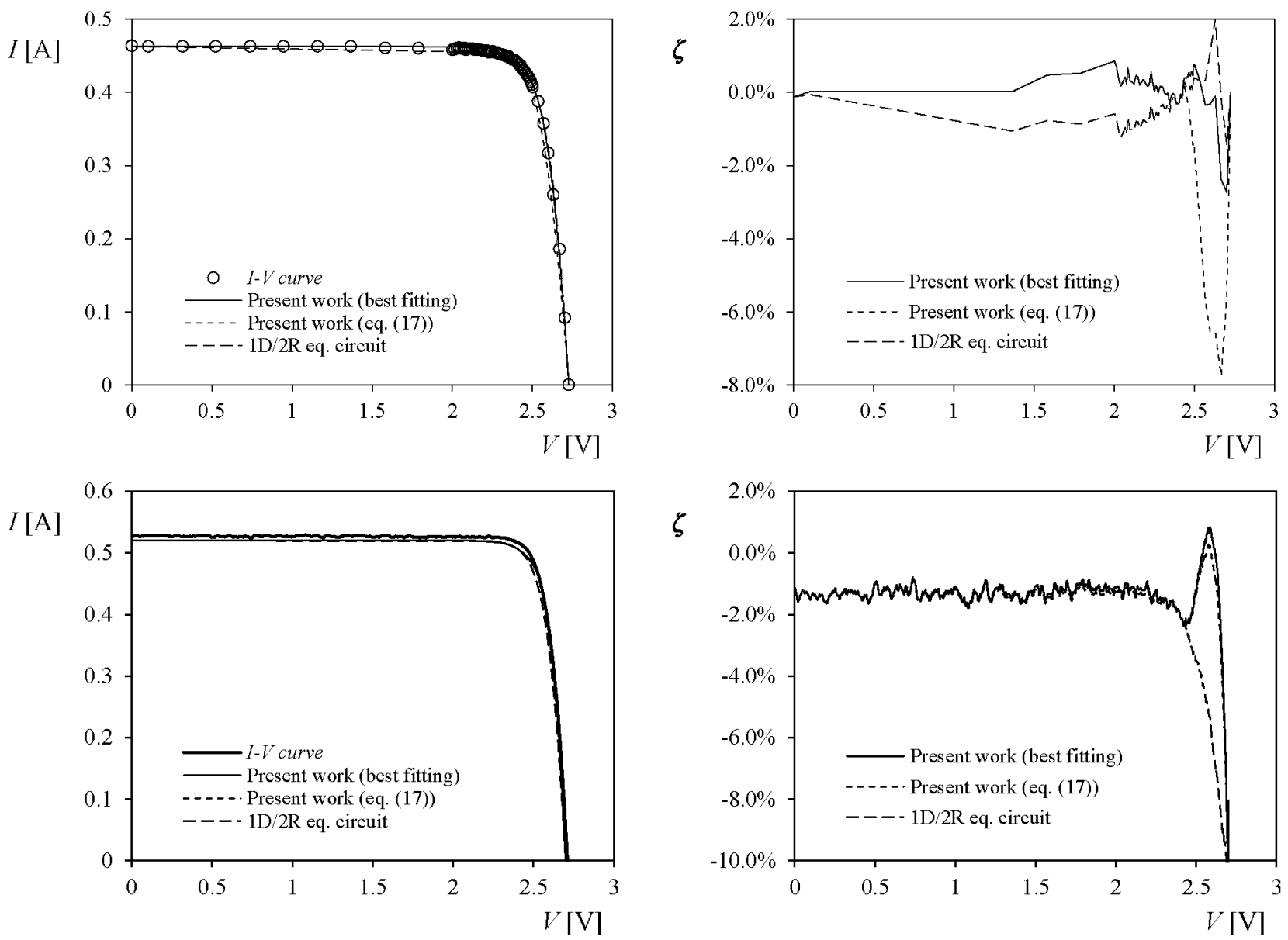

Fig. 7. On the left graphs: I-V curves modeled with the present approach and the 1-diode/2-resistor equivalent circuit model fitted to ZTJ Emcore (top) and Azur Space 3G30C (bottom) solar cell reference curves The reference curves are also included in the graphs (labeled as $I-V$ curve). Due to the large number of points related to Azur Space $3 \mathrm{G} 30 \mathrm{C}$ solar cell reference curve, this curve is plotted with a solid line and instead with open circles. On the right graphs: differences between the studied models and the reference curves, made non dimensional with the short circuit current (Equation (40)).

the behavior of the photovoltaic devices included in Table 1 , as $I-V$ curves of these solar cells/panels were available.

$I-V$ curves of RTC France solar cell and Photowatt PWP 201 solar panel were obtained from Ref. [36], together with the characteristic points. $I-V$ curves of TNJ Spectrolab and ZTJ Emcore solar cells, and Kyocera KC200GT-2 solar panel were obtained graphically from the manufacturer's datasheet (their characteristic points were also obtained from the datasheets). I- $V$ curve of Azur Space 3G30C solar cells was kindly supplied by the manufacturer, the characteristic points being obtained from the datasheet. Finally, the $I-V$ curve of Selex Galileo SPVS X5 solar module was measured at CIEMAT (Spain), and the characteristic points at testing temperature, $T=20.12{ }^{\circ} \mathrm{C}$, were derived from the characteristic points at $T=28{ }^{\circ} \mathrm{C}$ obtained in the cells manufacturer's datasheet (Azur Space $3 \mathrm{G}-28 \%$ ). All this curves will be referred as $I-V$ reference curves hereinafter.

In Figs. $6-8$, the reference $I-V$ curves of each photovoltaic device are compared to:

- two I-V curves obtained with the proposed mathematical model (one calculated with the constant $\eta$ that gives the best fitting within the second bracket, $\left[V_{m p}, V_{o c}\right]$, and the other one calculated with the constant $\eta$ estimation based on Equation (21)), and

- $I-V$ curve from the 1-diode/2-resistor equivalent circuit model, calculated analytically with the corresponding characteristic points.

Additionally, calculated values of constant $\eta$ used for the present mathematical approach within the second bracket $\left[V_{m p}, V_{o c}\right]$, are included in Table 2 for all studied photovoltaic devices. This information reveals the accuracy of the proposed Equation (21) to calculate this constant. The parameters related to the 1 -diode/2resistor equivalent circuit model (Equation (1)) are included in Table 3. The parameter extraction was performed as explained in Ref. [2]. An initial estimation of the ideality factor $a=1.2$, was modified after a few iterations to obtain a better fitting to the $I-V$ reference curve.

A similar correlation with the reference data of both, the curves based on the proposed methodology and the curve based on the 1diode/2-resistor equivalent circuit model, can be observed in the graphs related to the $I-V$ curves included in the aforementioned figures. Besides, the difference between the output current calculated with models and the one from the reference data, related to the short circuit current: 

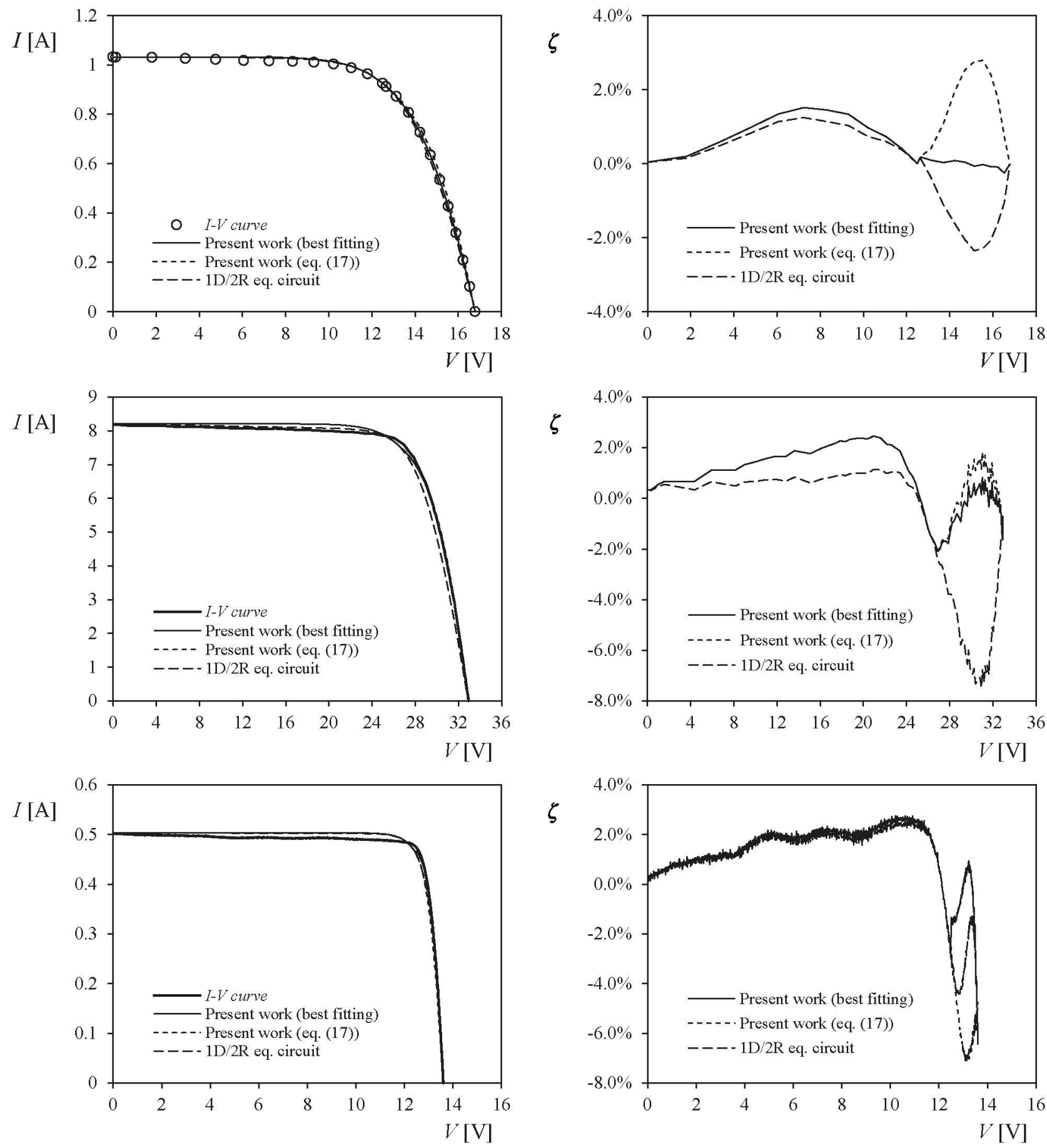

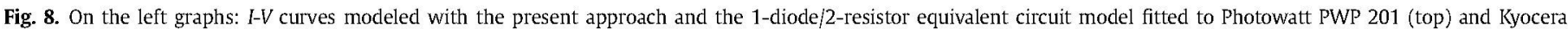

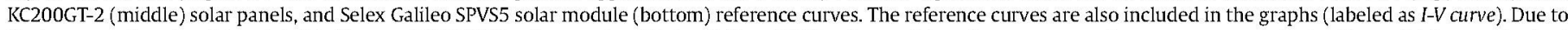

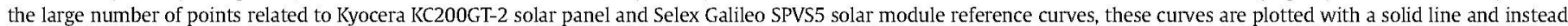

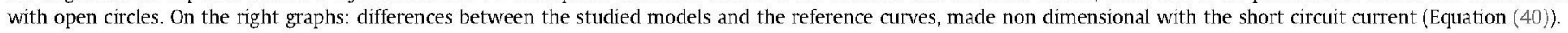


Table 2

Values of the present model constant $\eta$ (bracket $\left[V_{m p}, V_{o c}\right]$ ), calculated for the studied photovoltaic devices with the different approaches suggested in the text.

\begin{tabular}{llllll}
\hline Photovoltaic device & $\eta_{b f}$ & $\eta_{p}$ & $\eta_{s l}$ & $\eta_{\text {es } 1}$ & $\eta_{\text {es } 2}$ \\
\hline RTC France & 2.52 & 2.50 & 2.48 & 2.67 & 2.51 \\
TNJ Sp-10ctrolab & 3.56 & 3.20 & 2.36 & 1.23 & 2.28 \\
ZTJ Encore & 2.90 & 2.94 & 3.16 & 1.24 & 2.37 \\
Azur Space 3G30C & 3.72 & 3.63 & 3.06 & 1.11 & 3.63 \\
Photowatt-PWP 201 & 2.48 & 2.48 & 2.47 & 3.42 & 2.76 \\
Kyocera KC200GT-2 & 2.85 & 2.90 & 3.63 & 2.42 & 2.96 \\
Selex Galileo SPVS5 & 3.85 & 3.86 & 3.15 & 1.13 & 3.04 \\
\hline
\end{tabular}

complex. Also, the proposed methodology to calculate the $\eta$ constant based on the $I-V$ curve slope estimation at the open circuit point (Equation (21)), produce a quite reasonable fitting of the approach to the $I-V$ curve, with a lower accuracy level when compared to the 1-diode/2-resistor model, but being of the same order of magnitude. Finally, less accurate results are obtained in the second bracket if constant $\eta$ is calculated with the slope at open point estimated with Equation (17), proposed by Orioli and Gangi [31].

Table 3

Parameters calculated for the 1-diode/2-resistor equivalent circuit model in relation to the studied photovoltaic devices.

\begin{tabular}{|c|c|c|c|c|c|c|c|}
\hline Photovoltaic device & $n$ & $T\left[{ }^{\circ} \mathrm{C}\right]$ & $a$ & $R_{s}[\Omega]$ & $R_{s h}[\Omega]$ & $I_{0}[\mathrm{~A}]$ & $I_{p v}[\mathrm{~A}]$ \\
\hline RTC France & 1 & 33 & 1.48 & $3.62 \cdot 10^{-2}$ & $5.20 \cdot 10^{1}$ & $3.20 \cdot 10^{-7}$ & $7.61 \cdot 10^{-1}$ \\
\hline TNJ Spectrolab & 3 & 28 & 1.01 & $5.51 \cdot 10^{-2}$ & $2.08 \cdot 10^{2}$ & $3.49 \cdot 10^{-15}$ & $5.24 \cdot 10^{-1}$ \\
\hline Azur Space $3 \mathrm{G} 30 \mathrm{C}$ & 3 & 28 & 0.9 & $7.95 \cdot 10^{-2}$ & $2.62 \cdot 10^{3}$ & $9.55 \cdot 10^{-18}$ & $5.20 \cdot 10^{-1}$ \\
\hline Photowatt PWP 201 & 36 & 45 & 1.25 & 1.56 & $3.55 \cdot 10^{3}$ & $1.28 \cdot 10^{-6}$ & 1.03 \\
\hline Kyocera KC200GT-2 & 54 & 25 & 1 & $3.36 \cdot 10^{-1}$ & $1.59 \cdot 10^{2}$ & $4.03 \cdot 10^{-10}$ & 8.23 \\
\hline Selex Galileo SPVS5 & 15 & 20 & 1.15 & $2.40 \cdot 10^{-2}$ & $9.69 \cdot 10^{3}$ & $1.48 \cdot 10^{-14}$ & $5.03 \cdot 10^{-1}$ \\
\hline
\end{tabular}

Table 4

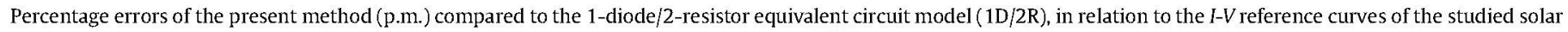
cells/panels. Errors calculated for the two brackets, $\left[0, V_{m p}\right]$ and $\left[V_{m p}, V_{o c}\right]$, with Equation (22).

\begin{tabular}{|c|c|c|c|c|c|c|c|c|}
\hline \multirow[t]{2}{*}{ Photovoltaic device } & \multicolumn{2}{|c|}{ First bracket, $\left[0, V_{m p}\right]$, } & \multicolumn{6}{|c|}{ Second bracket, $\left[V_{m p}, V_{o c}\right]$} \\
\hline & $1 \mathrm{D} / 2 \mathrm{R}$ & p.m. & $1 \mathrm{D} / 2 \mathrm{R}$ & p.m. $\left(\eta_{b f}\right)$ & p.m. $\left(\eta_{p}\right)$ & p.m. $\left(\eta_{s i}\right)$ & p.m. $\left(\eta_{e s i 1}\right)$ & p.m. $\left(\eta_{\text {esi2 }}\right)$ \\
\hline RTC France & $0.10 \%$ & $0.52 \%$ & $0.13 \%$ & $0.41 \%$ & $0.76 \%$ & $1.17 \%$ & $2.40 \%$ & $0.59 \%$ \\
\hline TNJ Spectrolab & $0.49 \%$ & $0.45 \%$ & $5.90 \%$ & $3.84 \%$ & $5.76 \%$ & $13.51 \%$ & $32.51 \%$ & $14.43 \%$ \\
\hline ZTJ Emcore & $0.72 \%$ & $0.31 \%$ & $1.01 \%$ & $0.50 \%$ & $0.57 \%$ & $1.78 \%$ & $20.97 \%$ & $4.42 \%$ \\
\hline Azur Space 3G30C & $1.38 \%$ & $1.30 \%$ & $6.58 \%$ & $1.48 \%$ & $1.66 \%$ & $5.81 \%$ & $37.84 \%$ & $1.64 \%$ \\
\hline Photowatt-PWP 201 & $0.78 \%$ & $0.97 \%$ & $4.73 \%$ & $0.21 \%$ & $0.28 \%$ & $0.42 \%$ & $17.04 \%$ & $5.44 \%$ \\
\hline Selex Galileo SPVS5 & $12.67 \%$ & $13.08 \%$ & $20.96 \%$ & $9.97 \%$ & $10.09 \%$ & $25.65 \%$ & $67.01 \%$ & $27.86 \%$ \\
\hline
\end{tabular}

$\zeta=\frac{I-I_{r e f}}{I_{s c}}$

has been included in Figs. 6-8 as a function of the output voltage (right graphs). In the first bracket $\left[0, V_{m p}\right]$, the present model is less accurate that the 1-diode/2-resistor equivalent circuit model. However, the accuracy in relation to the reference curves seems reasonable, around $2 \%$ of the short circuit current whereas the 1 diode/2-resistor model shows $1 \%$ accuracy. Focusing on the second bracket, the accuracy showed by the results of both models is much worse.

In order to quantify and compare the models, the percentage errors related to the $I-V$ reference curves were calculated with Equation (22) in both brackets, $\left[0, V_{m p}\right]$ and $\left[V_{m p}, V_{o c}\right]$. These results have been included in Table 4 . In the second bracket the error was calculated taking into account points within $\left[V_{m p}, 0.975 \cdot V_{o c}\right.$, that is, leaving aside those points very close to the open circuit voltage (as they imply a negligible current that leads the principal term of Equation (22) to extremely high figures, making the comparison between the present method and the equivalent circuit method impossible near the open circuit point).

Taking into account the figures of Table 4, it can be said that the proposed mathematical approach to the $I-V$ curve ( $\eta$ constant calculated with the best fitting, with one point within $\left[V_{m p}, V_{o c}\right]$, or with the slope of the $I-V$ curve at the open circuit point), has an accuracy level quite similar to the one related to the 1-diode/2resistor model, the use of the proposed approach being less

\section{Conclusions}

In the present work a new explicit mathematical expression is proposed to describe the behavior of a solar cell/panel, based on the three characteristic points of the $I-V$ curve. The major conclusions resulting from this work are:

- The proposed explicit equations, once properly adjusted, fit the behavior of the panel as well as the 1-diode/2-resistor equivalent circuit model.

- This methodology is simpler than other mathematical methods developed previously by other researchers, as it depends on one constant estimation only.

- A new equation to estimate the slope of the $I-V$ curve at the open circuit point has also been proposed, with reasonable results for the technologies studied ( $\mathrm{Si}$, triple junction).

\section{Acknowledgements}

The authors are indebted to José Lorenzo Balenzategui, from CIEMAT (Centro de Investigaciones Energéticas, Medioambientales y Tecnológicas), for his friendly and constant support in relation to measurements involving photovoltaic devices and sensors. Authors are also grateful to Azur Space for the kind support. Finally, authors are grateful to the Reviewers for their wise comments, which helped to improve this work. 


\section{References}

[1] J. Cubas, S. Pindado, C. de Manuel, Explicit expressions for solar panel equivalent circuit parameters based on analytical formulation and the lambert W-Function, Energies 7 (2014) 4098-4115.

[2] J. Cubas, S. Pindado, M. Victoria, On the analytical approach for modeling photovoltaic systems behavior, J. Power Sources 247 (2014) 467-474.

[3] D.T. Cotfas, P.A. Cotfas, S. Kaplanis, Methods to determine the dc parameters of solar cells: a critical review, Renew. Sustain. Energy Rev. 28 (2013) 588-596.

[4] A.K. Tossa, Y.M. Soro, Y. Azoumah, D. Yamegueu, A new approach to estimate the performance and energy productivity of photovoltaic modules in real operating conditions, Sol. Energy 110 (2014) 543-560.

[5] A. Ortiz-Conde, F.J. García-Sánchez, J. Muci, A. Sucre-González, A review of diode and solar cell equivalent circuit model lumped parameter extraction, FACTA Univ. Ser. Electron. Energy 27 (2014) 57-102.

[6] D. Jena, V.V. Ramana, Modeling of photovoltaic system for uniform and nonuniform irradiance: a critical review, Renew. Sustain. Energy Rev. 52 (2015) 400-417.

[7] V.J. Chin, Z. Salam, K. Ishaque, Cell modelling and model parameters estimation techniques for photovoltaic simulator application: a review, Appl. Energy 154 (2015) 500-519.

[8] A.M. Humada, M. Hojabri, S. Mekhilef, H.M. Hamada, Solar cell parameters extraction based on single and double-diode models: a review, Renew. Sustain. Energy Rev. 56 (2016) 494-509.

[9] F.J. Toledo, J.M. Blanes, A. Garrigós, J.A. Martínez, Analytical resolution of the electrical four-parameters model of a photovoltaic module using small perturbation around the operating point, Renew. Energy 43 (2012) 83-89.

[10] F.J. Toledo, J.M. Blanes, Geometric properties of the single-diode photovoltaic model and a new very simple method for parameters extraction, Renew. Energy 72 (2014) 125-133.

[11] F.J. Toledo, J.M. Blanes, Analytical and quasi-explicit four arbitrary point method for extraction of solar cell single-diode model parameters, Renew. Energy 92 (2016) 346-356.

[12] M. Green, Accuracy of Analytical Expressions for Solar-cell Fill Factor, 1982.

[13] R.A.H. Khanna, T. Stangl, B. Basu, P.K. Aberle, A.G.A. Mueller, A. Khanna, T. Mueller, R.A. Stangl, B. Hoex, P.K. Basu, et al., A fill factor loss analysis method for silicon wafer solar cells, IEEE J. Photovolt. 3 (2013) 1170-1177.

[14] M.A. Green, Accurate expressions for solar cell fill factors including series and shunt resistances, Appl. Phys. Lett. 108 (2016) 1-5.

[15] J. Cubas, S. Pindado, A. Farrahi, New method for analytical photovoltaic parameter extraction, in: Proceeding of 2nd International Conference on Renewable Energy Research Application ICRERA 2013, IEEE Press, Madrid, Spain, 2013, pp. 873-877.

[16] J. Cubas, S. Pindado, C. De Manuel, New method for analytical photovoltaic parameters identification: meeting Manufacturer's datasheet for different ambient conditions, in: A.Y. Oral, Z.B. Bahsi, M. Ozer (Eds.), International Congress on Energy Efficiency Energy Related Materials (ENEFM2013), Springer Proceeding in Physics 155, Springer International Publishing, Antalya, Turkey, 2014, pp. 161-169.

[17] C. Manuel, J. Cubas, S. Pindado, On the simulation of the UPMSat-2 microsatellite power, in: European Spac Power Conference ESA, vol. 2014, ESA, April
15-17, 2014, pp. 1-7.

[18] S. Pindado, J. Cubas, F. Sorribes-palmer, On the analytical approach to present engineering Problems: photovoltaic systems behavior, wind speed sensors performance, and high-speed train pressure wave effects in tunnels, Math. Probl. Eng. 2015 (2015) 1-17.

[19] M. Akbaba, M.A.A. Alattawi, A new model for I-V characteristic of solar cell generators and its applications, Sol. Energy Mater. Sol. Cells 37 (1995) $123-132$.

[20] R. Miceli, A. Orioli, A. Di Gangi, A procedure to calculate the I-V characteristics of thin-film photovoltaic modules using an explicit rational form, Appl. Energy 155 (2015) 613-628.

[21] A. El Tayyan, An empirical model for generating the IV characteristics for a photovoltaic system, J. Al-Aqsa Unv. 10 (2006) 214-221.

[22] A.A. El Tayyan, A simple method to extract the parameters of the single-diode model of a PV system, Turk. J. Phys. 37 (2013) 121-131.

[23] W. Xiao, M.G.J. Lind, W.G. Dunford, A. Capel, Real-time identification of optimal operating points in photovoltaic power systems, IEEE Trans. Ind. Electron 53 (2006) 1017-1026.

[24] L. Peng, Y. Sun, Z. Meng, Y. Wang, Y. Xu, A new method for determining the characteristics of solar cells, J. Power Sources 227 (2013) 131-136.

[25] S. Karmalkar, S. Haneefa, A physically based explicit J-V model of a solar cell for simple design calculations, IEEE Electron Device Lett. 29 (2008) 449-451.

[26] S. Haneefa, S. Kamalkar, An analytical method to extract the physical parameters of a solar cell from four points on the illuminated J-V curve, Electron Device Lett. IEEE 30 (2009) 349-352.

[27] A.K. Das, An explicit J-V model of a solar cell for simple fill factor calculation, Sol. Energy 85 (2011) 1906-1909.

[28] T.O. Saetre, O.M. Midtgard, G.H. Yordanov, A new analytical solar cell I-V curve model, Renew. Energy 36 (2011) 2171-2176.

[29] A.K. Das, An explicit J-V model of a solar cell using equivalent rational function form for simple estimation of maximum power point voltage, Sol. Energy 98 (2013) 400-403.

[30] P. Bhatnagar, R.K. Nema, Maximum power point tracking control techniques: state-of-the-art in photovoltaic applications, Renew. Sustain. Energy Rev. 23 (2013) 224-241.

[31] A. Orioli, A. Di Gangi, A procedure to calculate the five-parameter model of crystalline silicon photovoltaic modules on the basis of the tabular performance data, Appl. Energy 102 (2013) 1160-1177.

[32] M.H. Deihimi, R.A. Naghizadeh, A.F. Meyabadi, Systematic derivation of parameters of one exponential model for photovoltaic modules using numerical information of data sheet, Renew. Energy 87 (2016) 676-685.

[33] J.C.H. Phang, D.S.H. Chan, J.R. Phillips, Accurate analytical method for the extraction of solar cell model parameters, Electron. Lett. 20 (1984) 406-408.

[34] R. Chenni, M. Makhlouf, T. Kerbache, A. Bouzid, A detailed modeling method for photovoltaic cells, Energy 32 (2007) 1724-1730.

[35] G.L. Araujo, E. Sanchez, A new method for experimental determination of the series resistance of a solar cell, Electron Devices IEEE Trans. ED 29 (1982) $1511-1513$.

[36] T. Easwarakhanthan, J. Bottin, I. Bouhouch, C. Boutrit, Nonlinear minimization algorithm for determining the solar cell parameters with microcomputers, Int. J. Sol. Energy 4 (1986) $1-12$. 